\title{
L'CEuvre ouverte. Michel Jeanneret ou la perpétuelle mobilité des formes
}

\section{Frank Lestringant, Frédéric Tinguely et Dominique Brancher}

\section{(2) OpenEdition}

1 Journals

\section{Édition électronique}

URL : https://journals.openedition.org/studifrancesi/20634

DOI : 10.4000/studifrancesi.20634

ISSN : 2421-5856

Éditeur

Rosenberg \& Sellier

\section{Édition imprimée}

Date de publication : 1 décembre 2019

Pagination : 531-542

ISSN : 0039-2944

\section{Référence électronique}

Frank Lestringant, Frédéric Tinguely et Dominique Brancher, «L'(Euvre ouverte. Michel Jeanneret ou la perpétuelle mobilité des formes », Studi Francesi [En ligne], 189 (LXIII | III) | 2019, mis en ligne le 01 décembre 2020, consulté le 12 novembre 2021. URL : http://journals.openedition.org/studifrancesi/ 20634 ; DOI : https://doi.org/10.4000/studifrancesi.20634

\section{(c) (i) $\odot$}

Studi Francesi è distribuita con Licenza Creative Commons Attribuzione - Non commerciale - Non opere derivate 4.0 Internazionale. 


\title{
L'OEuvre ouverte. Michel Jeanneret ou la perpétuelle mobilité des formes
}

\begin{abstract}
As a tribute to Michel Jeanneret, who died on 3 March 2019, three of his friends, Dominique Brancher, Frank Lestringant and Frédéric Tinguely, recall here the three periods of his output as a literary critic, which span the period between the Renaissance and the Romantics, deliberately rendering permeable the borders which separate centuries and disciplines. In turn, are considered the Renaissance with its shifting boundaries, the different characteristics of the Grand Siècle, and then folly in its different forms, from Erasmus to Nerval. For Michel Jeanneret, criticism was not an exercise confined to a study or relegated within the four walls of a classroom, but was an activity open to life, one which radiates outwards, questioning the present and laying foundations for the future. It was not an activity confined to a particular century, but was always overflowing - and in all directions - out of the constraining compartmentalisation of our specialist fields. For him, the Renaissance was only a point of departure, a mobile base out of which opened broad avenues sweeping through, and almost rending apart, the Classical age, while charting fruitful and at times disturbing paths forward through to our modern day. In the tight fabric of our literary history, Michel Jeanneret has opened up a fertile space, a wide clearing which we here attempt to explore.
\end{abstract}

\section{Une Renaissance mobile}

Comment attraper la Renaissance ou la prendre, sans qu'elle se débatte? Ou, pour emprunter une image plus sereine, plus convenue et assurément moins sexiste, comment faire de la Renaissance une peinture en mouvement, inachevée, bariolée, fidèle jusque dans ses intermittences à son objet précaire, éminemment instable?

On retiendra ici deux livres de Michel Jeanneret, Des mets et des mots. Banquets et propos de table à la Renaissance ${ }^{1}$, et Perpetuum mobile. Métamorphoses des corps et des œuvres de Vinci à Montaigne ${ }^{2}$, auxquels s'ajoute pour mémoire, et pour le plaisir des yeux, et presque de la bouche, La Renaissance italienne à pleines dents, présence charnelle surgie du plat et à portée de lèvres 3 .

Des mets et des mots pour commencer. Ce livre marque une rupture par rapport à la thèse sévère qui portait sur les paraphrases des psaumes de Marot à Malherbe, mais sans reniement, dans l'insensible continuité qui conduit des mots aux choses, choses le plus souvent savoureuses et délicieuses ${ }^{4}$. Parler en mangeant, absorber

(1) M. Jeanneret, Des mets et des mots. Banquets et propos de table à la Renaissance, Paris, José Corti, 1987.

(2) M. Jeanneret, Perpetuum mobile. Métamorphoses des corps et des ouvres de Vinci à Montaigne, $2^{\mathrm{e}}$ édition revue et complétée d'une postface, Genève, Droz, 2016, «Titre courant». Ce livre a d'abord paru en 1997 dans la collection Argô de Macula.

(3) Fondation Barbier-Mueller, La Renaissance italienne à pleines dents, édité par N. Ducimetière et M. Jeanneret, Paris, Somogy-Éditions d'art, 2016, pp. 7-13.

(4) M. Jeanneret, Poésie et tradition biblique au XVI siècle. Recherches stylistiques sur les paraphrases des psaumes de Marot à Malherbe, Paris, Librairie José Corti, 1969. 
tout en proférant, comment ces deux trajets, en apparence contraires, peuvent-ils se concilier, à moins de s'étrangler?

Le symposiaque, dira-t-on, les joint dans une même célébration. Oui, mais qu'entendre par là? Le symposiaque, c'est tout simplement le fait de boire et de dire, de manger en parlant, de mêler la parole à la nourriture, solide et liquide. Le symposiaque, loin de tout tohu-bohu bachique, poursuit l'«idéal d'une parole digeste» ${ }^{5}$. Il fait de la langue cet organe souple et réversible, capable tour à tour d'avaler et de proférer, pour savourer et dire élégamment, prestement, au proche voisin aussi bien qu'à l'ensemble des convives. De sorte que «langue mangeante et langue parlante» entretiennent, dans leurs phases alternées, une rigoureuse continuité ${ }^{6}$. Les mets et les mots sont solidaires, quoiqu'ils remplissent la bouche alternativement pour déglutir et pour dire.

La voie royale est bien sûr Rabelais, avec ses franches repaissailles, ses banquets festifs qui accompagnent la naissance du héros Gargantua, aussi bien que les principaux épisodes de la guerre picrocholine. Le vin coule à flots, submergeant le sang des vaincus. Mais l'on pense aussi à Ronsard et à Montaigne. Ronsard, tout d'abord, lecteur de l'Anthologie grecque, poète en état d'ébriété bachique quand il compose les Odes, ou plus tard la Franciade, même si la parole, alors, succède sagement à la nourriture, sans plus se mêler à elle. Montaigne, dans les Essais, dresse le bilan, «quelque peu tératologique», des abus de l'imitation ${ }^{7}$. Comme il le confesse, Montaigne absorbe à plaisir et tout devient sien. Mais le moi rejaillit dans cette profération d'emprunt, tant il est vrai que le discours est une force en quête de forme, «une énergie que l'art n'a pas encore figée» ${ }^{8}$. L'œuvre ouverte et instable, le texte de la Renaissance n'est pas un monument accompli, «c'est un événement en cours de réalisation» ${ }^{9}$. Une ébauche, une bouche, encore chargée des vestiges de la déglutition, qui, bientôt, au siècle classique, va proférer, dans une orgueilleuse solitude, et dans une apparence de netteté impeccable. Telle sera la fiction classique du texte clos, parfait, éternel, une fiction qui tranche avec le texte ouvert, le texte mêlé de la Renaissance, comme le vin que l'on mêle à l'eau dans les cratères au temps des aèdes, au temps encore où les devisants s'allongeaient côte à côte pour converser joyeusement et pour boire.

Des mets et des mots précède de dix ans Perpetuum mobile dont il constitue en quelque sorte le prélude. D'emblée, Perpetuum mobile ne se limite pas au champ littéraire, parcouru des Adages d'Érasme aux Essais de Montaigne, et des Odes de Ronsard aux Tragiques d'Agrippa d'Aubigné. Très vite il s'élargit à la gravure, à la peinture, aux dessins fugaces de Léonard aussi bien qu'aux paysages anthropomorphes d'Athanase Kircher ou d'Arcimboldo ${ }^{10}$. Partout, la mobilité bat en brèche toute tentative d'arrêter le réel, de fixer le probable, de figer l'instable. La sensibilité métamorphique n'est sans doute pas le seul aspect de la Renaissance, mais c'en est l'aspect principal, prédominant, qui emporte le reste. «La force bouscule la forme, la variété supplante l'unité, Dionysos occulte Apollon» ${ }^{11}$. Cette bousculade de la phrase fait surgir la joie dionysiaque qui explose et irradie.

Comme le dit sagement l'introduction, qui, d'entrée de jeu, annonce qu'il n'y

(5) M. Jeanneret, Des mets et des mots cit., p. 90.

(6) Ibidem, p. 92.

(7) Ibidem, p. 265.

(8) Ibidem, p. 268.

(9) Ibidem, p. 269.

(10) M. Jeanneret, Perpetuum mobile cit.

(11) Ibidem, p. 387. 
aura pas de conclusion: «on peut toujours commencer, on ne finit jamais» ${ }^{12}$. Commençons donc par le commencement. Au départ, une prolifération, tout simplement. La vie prolifère sans s'arrêter, sans s'interrompre, sans repentirs ni retours. La vie va de l'avant, et nous dépasse tous en tant qu'individus, quitte à nous déborder, nous emporter et parfois nous engloutir.

La littérature, on pourrait la croire enfermée dans des siècles, dans des genres, régie par des règles infrangibles. Mais la littérature s'enferme-t-elle dans des normes, dans des règles? La littérature ne risque-t-elle pas de mourir de sa belle mort, si on l'enferme, si on la règle, si on la borne? La littérature a-t-elle ses règles, ses lois, ses arrêts? Ainsi pensait-on les classiques au temps de notre adolescence. Le XVII siècle était le Grand Siècle, le siècle insurpassable, avec Corneille et Racine, encadrant sagement Molière. Tout ce qui avait précédé et avait suivi était secondaire, ravalé aux utilités. Le XIX ${ }^{\mathrm{e}}$ siècle avait tout juste droit à l'existence, avec Balzac, avec Stendhal, avec Flaubert, et surtout les poètes, de Hugo à Mallarmé, en passant par Verlaine et Rimbaud. Quant au Xx siècle, il était tout simplement absent, hormis Paul Valéry et le presque inconnu Henri Michaux, dont raffolait notre professeur de seconde. Hormis ces rares exceptions, on en revenait au Cid, à Horace, à Cinna, peut-être à Andromaque. Toujours la règle des trois unités garantissait la norme. On ne pouvait faire mieux, rêver mieux, oser mieux.

Michel Jeanneret a tenté de briser ce cadre rigide et de faire déborder cette Renaissance tumultueuse, infiniment diverse dans sa fugacité, sur le XVII siècle. La Renaissance se prolonge et déborde sur le siècle classique. Incandescente, folâtre, juvénile, et déjà en crise, malgré son nom, son programme, et quelques-unes de ses réalisations, la Renaissance s'ébroue, toute tachée de sang, et rebondit, subreptice, insistante, pour repartir, lançant un défi à l'ordre établi du Grand Siècle, à la sérénité de commande de l'écrasant Grand Siècle! D'où les malheureux libertins rabroués, censurés, condamnés, bientôt interdits, et la fin malheureuse de Théophile de Viau, menacé du bûcher, puis relâché après repentance et mourant peu après. D'où la solitude de Molière et les insondables tréfonds du Misanthrope et de Tartuffe, sans parler même de Dom Juan. Qu'en dire, qu'en penser aujourd'hui? Ouvrir et faire flotter, ouvrir et laisser l'ensemble partir à la dérive. Et observer ce qui peut advenir, deviner l'impulsion qui se prolonge par à-coups et fait trembler encore les marbres de Versailles ${ }^{13}$.

Revenons au XVI ${ }^{\mathrm{e}}$ siècle. Pourquoi ce siècle au détriment des autres? Pourquoi Michel Jeanneret a-t-il choisi la Renaissance comme période privilégiée? Avant de la faire déborder, jusqu'au siècle suivant, jusqu'au XVIII ${ }^{\mathrm{e}}$ siècle même et aux prodromes de la Révolution, bien présents dans l'alerte réplique de Suzanne à Figaro: «J'aime ta joie, parce qu'elle est folle» ${ }^{14}$. C'est que le $\mathrm{XVI}^{\mathrm{e}}$ siècle est «emporté par le changement» ${ }^{15}$, qu'il tressaille de cette mobilité incessante, en tous sens, en tous lieux, en toutes circonstances.

Tout commence, pourrait-on penser, par une stabilité apparente, avec Du Bartas et sa divine Sepmaine, auquel est consacré le premier chapitre ${ }^{16}$. Stabilité en vérité trompeuse, puisque la Création, quand bien même accomplie, bouge et ne cesse de

(12) Ibidem, p. 15.

(13) M. Jeanneret, Versailles, ordre et chaos, Paris, Gallimard, 2012. Voir ci-après F. Tinguely, «Toutes les saveurs du Grand Siècle».

(14) M. Jeanneret, J'aime ta joie parce qu'elle est folle. Écrivains en fête (XVI et XVII siècles), Genève, Droz, «Titre courant», 2018. La réplique est celle de Suzanne à Figaro, in Beaumarchais, Le Mariage de Figaro, acte IV, scène 1: «J'aime ta joie, parce qu'elle est folle; elle annonce que tu es heureux».

(15) M. Jeanneret, Perpetuum mobile cit., p. 7.

(16) Ibidem, pp. 19-40: «La forme et la force: Du Bartas». 
se renouveler, en dépit de l'ordre immuable de Dieu. Création continue, sans aucun doute, ou plutôt Création continuée, la leçon de Lucrèce se mariant à celle de la Bible, pour faire bouger l'apparence étale des choses. Même Luther concède une place à l'évolution, comme plus tard Bernard Palissy, pour lequel «la mer se promène d'un côté et d'autre, et se travaille à produire choses profitables».

Cette image d'un monde flexible, d'un moi incessamment divers comme le monde où il se coule, peut revêtir des apparences plus joyeuses, comme chez Ronsard, qui éprouve la dilatation et la dispersion comme «l'occasion, ludique et joyeuse, d'explorer différents modes d'être» ${ }^{17}$. Avant la reprise en mains qui suivra le concile de Trente, une pensée syncrétique se met en place, qui libère le verbe, laissant toute la place au dynamisme de la parole, débordante, effulgente, ravissante.

Venons-en aux dessins de Léonard, brouillons, inventifs, multipliant les monstres à voir plutôt qu'à redouter, alignant les faciès hostiles ou vieillissants, représentant les mouvements insidieux de l'eau, «puissance molle» ${ }^{18}$, imaginant les affres du déluge qui submerge tout, dans une indifférenciation généralisée. Sans parler des «cartes mobiles» ${ }^{19}$, cartes d'un monde incomplet, en devenir, qui s'adaptent à toute nouvelle découverte, notamment au sud, en cette « terre australe » que le cartographe Guillaume Le Testu déploie de Java à la Terre de feu, en attendant que connaissance certaine en soit donnée ${ }^{20}$. Puis viennent les Trois Mondes que prophétise le protestant La Popelinière: à l'ancien et au nouveau mondes, déjà connus, s'en ajoute un troisième, qu'il reste à découvrir. Il suffit d'aller vers le sud. Là s'étendra un empire qu'il appartient à la France de fonder. Avant même d'exister, voici ce troisième monde cartographié par Abraham Ortelius dans son Theatrum orbis terrarum, une vaste terre australe qui forme contrepoids aux continents de l'hémisphère boréall ${ }^{21}$.

Monde mobile, monde instable, guère plus improbable que ne l'était le Nouveau Monde à présent connu de tous, mais qui recèle encore bien des béances. Témoignent encore de cette instabilité, de cette incomplétude, de ce non finito, le jardin de Bomarzo, peuplé de statues inquiétantes, à demi arrachées au sol, et le géant Apennin surgi au milieu du parc de Pratolino, montagne à demi vivante, figée depuis cinq siècles par le marteau du sculpteur Giambologna, et surtout les ténébreuses grottes qui s'entrouvrent, remplies d'invraisemblables grotesques. D'origine satanique, indiscutablement, selon les théologiens inquiets, voire horrifiés, de la Contre-Réforme, ces grotesques, mi-hommes mi-bêtes, mi-créatures mi-abstractions géométriques, prolifèrent et se répandent.

Mais pourquoi s'inquiéter de cette incessante plasticité? L'homme peut au contraire en tirer gloire, comme Pic de La Mirandole dans son éloge de l'inventivité humaine. L'Oratio de hominis dignitate, l'un des manifestes les plus vigoureux de la pensée humaniste, propose au lecteur, à l'interlocuteur, serait-on tenté de dire, de se modeler lui-même, à l'exemple d'une nature débordante et malléable. Érasme, l'infatigable compilateur des Adages, lesquels se regroupent bientôt par «chiliades», c'est-à-dire par milliers, alignés et commentés dans un ordre aléatoire, au hasard de

(17) Ibidem, p. 57

(18) Ibidem, p. 81

(19) Ibidem, p. 93.

(20) Guillaume Le Testu, Cosmographie Universelle. Selon les navigateurs tant anciens que modernes par Guillaume Le Testu pillotte en la mer du Ponent, de la ville françoyse de Grace, présentation de F. Lestringant, Paris, Arthaud, Direction de la Mémoire, du Patrimoine et des Archives, Carnets des Tropiques, 2012 .

(21) Henri de La Popelinière, Les Trois Mondes de La Popelinière, 1582, Genève, Droz, 1997, p. 109. La carte du monde est naturellement empruntée à Abraham Ortelius, Theatrum orbis terrarum, Anvers, 1570. 
l'enquête dans les livres imprimés ou manuscrits, grecs ou latins, a, sa vie durant, remis sur le métier ses glanes incessantes, toujours augmentées.

Rien n'est jamais fixé! Tout reprend ou redémarre, à chaque nouvelle impression, de sorte que le livre imprimé ne fixe pas définitivement l'essai, qui reste un essai, une tentative incomplète et toujours à reprendre. Â preuve, Montaigne dont les Essais prolifèrent, sans cesse en mouvement, en croissance - croissance retorse, non pas linéaire, mais turbulente, revenante, insistante, qui se contredit elle-même et se prend en flagrant délit d'inconstance.

On sait l'inclination de Montaigne pour la variété. Pour ce dernier, notre inconstance foncière rend toute synthèse impossible, d'où la mise en liste qu'il suggère. Puisqu'aucune démonstration n'est possible pour ce sceptique, place à l'énumération incongrue, au hors-sujet, à l'incohérence. La «mise en rôle» n'est pas une solution de facilité, c'est la seule solution possible. Et tout naturellement Les Essais, au sens de «coups d'essai», sont la conséquence de cette errance discursive, le sujet se décrivant, ou plutôt décomposant sa physionomie, ses goûts, ses appréhensions en liste cocasse, incohérente parfois, au fur et à mesure qu'ils lui viennent à l'esprit.

L'imprimé ne donne pas une leçon définitive, qui serait gravée à jamais. S’il tend à fixer le texte par rapport au manuscrit, dont la linéarité malléable se resserre et se stabilise en apparence, les différents exemplaires d'un tirage varient: repentirs du typographe, pages recomposées en fonction d'un accident de tirage, retouches qui sont parfois le fait de l'auteur, à moins que la jurisprudence ne s'en mêle, et que le texte d'un feuillet ne soit recomposé par décret de justice ${ }^{22}$. Le texte bouge, de la façon la plus expresse, en dépit des progrès mécaniques de l'art typographique.

L'invention littéraire tient compte de cette instabilité. L'une des formes les plus goûtées est le recueil de nouvelles, qui, du Décaméron aux Serées de Guillaume Bouchet et de l'Heptaméron de Marguerite de Navarre au Moyen de parvenir de Béroalde de Verville, multiplie les incidentes, les histoires à part, le style à part, le montage incongru. Aucune œuvre n'est définitive ni achevée, aucune n'interdit des prolongements, des suites, des reprises. Lire Rabelais, c'est traverser «une terre linguistique meuble, c'est capter les métamorphoses d'un corps malléable ${ }^{23}$. Rabelais n'est-il pas «l'un des fomentateurs de la crise herméneutique à la Renaissance ${ }^{24}$, partisan d'une pluralité de sens, mais soudain, sans avertissement, d'une perspective contraignante, qu'il assène avec force dans la dédicace du Quart Livre. Souvent «la lecture vacille dans un tourniquet de contradictions ${ }^{25}$. L'auteur est certes un augmentateur, comme le veut l'étymologie $e^{26}$, mais loin de recopier platement, il transforme ce qu'il emprunte, il en mêle les bribes et les fait siennes. Ainsi Montaigne jette des idées en cours de gestation, amorce des développements qui finissent en queue de poisson, laissant au lecteur une vaste réserve de biens à exploiter.

Dans leur surcharge de sens et d'intentions, les œuvres de la Renaissance sont des mobiles ${ }^{27}$. Prenons les statues inachevées de Michel-Ange, les quatre Esclaves d'abord destinés au tombeau de Jules II et finalement intégrés par Bernardo Buon-

(22) Comme le veut l'exemple de François de Belleforest, Histoire des neuf Roys Charles de France, Paris, Pierre L'Huillier, 1568, pp. 639-640. Voir sur ce point F. Lestringant, André Thevet, cosmographe des derniers Valois, Genève, Droz, 1991, pp. 167-173.

(23) M. Jeanneret, Perpetuum mobile cit., p. 227.

(24) M. Jeanneret, Le défi des signes. Rabelais et la crise de l'interprétation à la Renaissance, Orléans, Paradigme, 1994.

(25) M. Jeanneret, Perpetuum mobile cit., p. 353.

(26) Ibidem, p. 304.

(27) Ibidem, p. 347. 
talenti au décor rustique d'une grotte artificielle dans le jardin Boboli, à Florence, où ils demeureront pendant trois siècles. La statuaire inachevée change de sens, elle ne représente plus le drame de la lutte entre l'esprit et la matière, mais le surgissement de la vie du sein du $\operatorname{chaos}^{28}$.

Déception peut-être, mais joie devant un savoir ouvert, proliférant, jamais arrêté, jamais conclu. Impression d'une promenade au jardin, par maint détour, retour et contour, sans jamais se redire ou se répéter, ni surtout se perdre et s'ennuyer, parcourir le monde comme un livre en mouvement, souple, infini, inclassable, toujours à reprendre, à rouvrir, à relire, pour se laisser surprendre et séduire.

Ce livre promenade, le plus complet et le plus étourdissant de ceux qu'ait écrits Michel Jeanneret, ne comporte pas de conclusion, mais s'achève - ou commence par une invite à relire, à reprendre, à poursuivre: «Le principe du texte flexible repose sur un acte de foi naturiste: il associe l'activité du penseur, du savant et de l'artiste aux grands rythmes qui scandent la vie des choses et président à leurs métamorphoses ${ }^{29}$. Le tout, sans surprise, est placé sous le signe de la métamorphose. On en revient à Ovide, d'où tout est parti et où tout recommence.

FRANK LESTRINGANT Sorbonne Université

\section{Toutes les saveurs du Grand Siècle}

La contribution de Michel Jeanneret à notre connaissance et à notre interprétation du $\mathrm{XVII}^{\mathrm{e}}$ siècle français doit être envisagée comme une réaction saine à l'encontre des lectures lénifiantes longtemps pratiquées par l'histoire littéraire et répercutées dans les manuels scolaires. Ramené généralement à quelques auteurs canoniques, réduit à son processus de standardisation de la langue, de l'esthétique et des mœurs, le Grand Siècle autorisé par certains milieux et certaines institutions apparaissait pour le moins étriqué, uniformisé, sans surprise: c'est avec une parfaite cohérence que Michel Jeanneret, au fil des livres et des éditions de textes, s'est élevé contre cette tendance historiographique qu'il assimilait volontiers à un processus de pasteurisation - métaphore lactée dont je ne puis m'empêcher de penser qu'elle contenait aussi une allusion souriante à l'austérité calviniste. Il s'agissait de redonner sa pleine envergure au Grand Siècle, de prendre en quelque sorte l'expression mythographique à la lettre (plutôt que de simplement la contester) afin d'élargir l'empan des phénomènes considérés, de se montrer capable d'embrasser ensemble, sans crainte des tensions ni des jalousies, la «Muse lascive» et la «Muse sacrée», pour reprendre les beaux titres des anthologies poétiques publiées en $2007^{30}$. En s'employant à restituer au XVII siècle sa pleine diversité, Michel Jeanneret inscrivait son travail dans le prolongement de celui de son maître Jean Rousset, dont le grand livre sur la littérature baroque avait redonné sa juste place à l'esthétique de l'inconstance et de l'ostentation, selon une visée historiographique explicitée en ces termes:

(28) M. Jeanneret, Perpetuum mobile cit., pp. 288-294.

(29) Ibidem, p. 358.

(30) La Muse sacrée, Anthologie de la poésie spirituelle française (1570-1630), Introduction, notes et lexique par T. Cave et M. Jeanneret, Paris, José Corti, 2007 [édition revue et corrigée de Métamorphoses spirituelles, Paris, José Corti, 1972]; La Muse lascive, Anthologie de la poésie érotique et pornographique française (1560-1660), Introduction, notes et lexique par M. Jeanneret, Paris, José Corti, 2007. 
On ne prétend pas substituer aux anciens principes d'explication un principe nouveau qui les rendrait caducs, mais plutôt déplacer la perspective, assouplir le schéma historique traditionnel et enrichir notre vision de l'époque d'un éclairage qui en marque mieux la complexité. Si les conclusions de cette étude étaient acceptées, le XVII e siècle «classique» n'en serait nullement obscurci ou diminué; mais il apparaîtrait moins homogène et moins linéaire; au lieu d'un siècle en évolution progressive et monochrome, on verrait se dessiner plusieurs XVII ${ }^{e}$ siècles parallèles, alternés ou entremêlés, au sein desquels on reconnaîtrait au Baroque la valeur d'un ferment actif et d'une composante nécessaire ${ }^{31}$.

Les mots de Jean Rousset s'appliquent parfaitement, pour ce qui est du XVII siècle, au projet ou plus exactement au programme de Michel Jeanneret: apporter un nouvel éclairage, compléter un tableau fragmentaire, introduire de la différence et de la complexité là où d'autres tendent à uniformiser les choses. Cela bien entendu sans polémiquer inutilement, sans nullement contester les connaissances acquises mais en se contentant plutôt, avec respect et élégance, de les enrichir. Parcourons ensemble, en trois étapes correspondant chacune à un ouvrage, cette trajectoire critique visant à rendre au Grand Siècle la variété de ses saveurs.

Le premier temps était sans doute le plus susceptible de «décoiffer quelques perruques», selon l'expression haute en couleur qu'affectionnait Michel Jeanneret. C'est en effet celui de la parution d'Éros rebelle. Littérature et dissidence à l'âge classique (Seuil, 2003), dédié justement à la mémoire de Jean Rousset, disparu un an plus tôt. Le livre participe du grand renouveau des études sur les libertins, mais y introduit aussi une sensibilité particulière: tandis que la plupart des chercheurs s'attachent à l'émergence d'une philosophie subversive, et veillent à bien distinguer (contrairement aux censeurs de l'époque) libertinage de pensée et libertinage de mœurs, Michel Jeanneret étudie la célébration d'Éros en suggérant qu'elle entraîne des conséquences philosophiques: «Et si le sexe aiguisait l'esprit? Tandis que les médecins et philosophes mécanistes séparent les opérations mentales de la sphère des sens, d'autres voient, au contraire, dans la recherche du plaisir un ressort qui stimule l'intelligence» (p. 179). Ou encore, à propos des auteurs du célèbre Parnasse satyrique de 1622: «Les poètes donnent ici raison à leurs accusateurs: libertinage de mœurs et libertinage de pensée s'appellent l'un l'autre; le mépris des prescriptions morales conduit à la contestation des choses sacrées» (p. 43). L'immense mérite de Michel Jeanneret, c'est d'abord de prendre au sérieux une littérature licencieuse souvent dénigrée (voire reléguée dans les enfers des bibliothèques) et de parvenir à lui donner un sens, une place dans l'histoire culturelle et intellectuelle du XVII siècle. Au fil de ses commentaires d'une grande virtuosité, le corpus érotique et pornographique qui prolifère au cours des années 1600-1620 (et dont La Muse lascive offre un aperçu substantiel) apparaît comme une puissante montée de sève, venue en droite ligne de la Renaissance, face au contrôle de plus en plus sévère que l'Autel et le Trône voudraient exercer sur l'individu et la société. La littérature résiste; elle est provocation, compensation et contrepoids, si bien que même un texte de facture didactique comme L'École des filles (1655) doit moins être compris dans la perspective foucaldienne d'une volonté de savoir qu'appréhendé dans toute sa charge pornographique, sanctionnée par la saisie et la destruction des exemplaires de la première édition. Le culte d'Éros ne doit d'ailleurs pas seulement affronter les contraintes de la censure; il est comme déterminé par les structures mêmes de la pensée à laquelle il s'oppose: grâce à son impeccable connaissance de l'érotisme cosmique et syncrétiste de la Renaissance, Michel Jean- 
neret dégage clairement, par contraste, le dualisme qui caractérise bien souvent la littérature licencieuse du XVII ${ }^{\mathrm{e}}$ siècle. Même si le discours sur le sexe produit toujours des effets de pensée, quelque chose s'est perdu, comme chez Descartes, de l'union heureuse de l'esprit et du corps, et tel poème morbide de Sigogne finit par ressembler au discours des hommes d'Église stigmatisant le corps et ses plaisirs. Ou comment la pornographie tend un miroir à la prédication... D’une certaine façon, c'est la répression du libertinage flamboyant - avec, en particulier, la condamnation de Théophile de Viau en 1625 - qui permet de sortir de ce face-à-face en obligeant les écrivains à adopter, du moins lorsqu'ils publient leurs œuvres, des stratégies d'esquive et de dissimulation. Si Michel Jeanneret n'examine pas celles-ci dans les textes libertins, il montre que l'obsession du sexe imprègne alors une littérature bien plus vaste qu'on ne pourrait le soupçonner et révèle par exemple, dans certains vers cornéliens, une récurrence de syllabes immondes à faire rougir Chimène. Du corpus non canonique de l'«offensive obscène» à l'obscénité latente du canon classique, Eros rebelle traverse le XVII siècle comme un faune danserait à Saint-Sulpice!

Plus sage, du moins en apparence, est le magnifique Versailles, ordre et chaos (Gallimard, 2012), auquel il convient de rattacher deux éditions de textes: non seulement, à l'évidence, Les Fêtes de Versailles d'André Félibien (Gallimard, 2012), mais aussi, et bien plus tôt, Les Amours de Psyché et de Cupidon de La Fontaine (Le Livre de Poche, 1991, avec la collaboration de Stefan Schoettke). En dépit de ce que pourrait suggérer son titre, l'ouvrage ne se limite en aucune façon à une monographie sur Versailles, mais propose, à partir d'une enquête sur le parc et ses dispositifs singuliers, une réinterprétation d'ensemble de l'esthétique du Grand Siècle et de son «moment classique» par excellence (env. 1660-1680). Michel Jeanneret s'attache à redonner à l'art de cette époque toute sa complexité et toute sa vigueur en révélant que sa face lumineuse est indissociable d'une part d'ombre, d'une face anxieuse que l'historiographie a eu tendance à négliger. L'idée n'est pas simplement que l'ordre côtoie le chaos, mais plutôt que l'un et l'autre sont étroitement solidaires, ainsi que l'exprime à merveille le mot-valise chaosmos, emprunté à Joyce et un temps considéré comme titre possible de l'ouvrage. Dans cette perspective, l'œuvre classique figure moins un état d'harmonie définitif qu'elle ne représente un processus toujours recommencé de lutte contre le désordre. Elle est inchoative, en mouvement, et sa beauté réside avant toute chose dans l'émergence d'une forme. Michel Jeanneret organise sa démonstration en trois ensembles d'analyses. Il propose tout d'abord à son lecteur une éblouissante promenade dans le parc de Versailles, moins celui des années 1680 que celui des origines, des genèses, où le souvenir du magma primordial est encore bien présent. Afin de ressusciter ce monde de l'ombre et des monstres, il réunit une riche documentation dans laquelle les projets de Le Brun occupent souvent une place centrale. L'étude de la grotte de Thétis, qui «cristallise l'esprit de Versailles dans ses jeunes années» (p. 63) avant d'être démolie en 1684 , est un moment particulièrement fort de ce travail de restitution. Avec pour guides Michel Jeanneret et André Félibien, le lecteur effectue le parcours initiatique qui était proposé aux premiers visiteurs de l'antre artificiel: «Si le promeneur, au moment de pénétrer dans la grotte, pouvait se croire précipité dans un fouillis, sombre et confus, de roche et d'eau, il découvrait peu à peu un ordre, une harmonie, si bien que, revivant le passage du chaos au cosmos, il rejoignait, là encore, le moment primordial de la création» (p. 69). Après l'examen des grottes, des fontaines ou des statues, l'enquête se concentre sur les fêtes et les spectacles (comédies-ballets, ballets de Cour, opéras) qui animèrent Versailles avec la somptuosité que l'on sait. Ce que dégage alors Michel Jeanneret, c'est la profonde cohérence de ces réjouissances à la fois entre elles et avec la symbolique du palais en général. Ses superbes pages sur le caractère nocturne des fêtes, qui visent à conjurer 
la nuit, à l'ensoleiller en quelque sorte, éclairent le lecteur comme autrefois les flambeaux ou les feux d'artifice tiraient de l'ombre les courtisans fascinés. Un nouveau regard est porté sur certaines comédies-ballets de Molière, comme Georges Dandin ou Monsieur de Pourceangnac, qui, au-delà de leur aspect farcesque, s'intègrent à l'esthétique d'ensemble en ce qu'elles mettent elles aussi en scène le triomphe sur le chaos originel. Qu'il s'agisse du parc ou des fêtes et des spectacles, Michel Jeanneret insiste sur le fait que les phénomènes analysés, s'ils se prêtent à une lecture politique, ne sauraient s'y réduire: ils sont par nature polysémiques et mettent en jeu des données anthropologiques plus profondes. C'est ce que montre la troisième partie de l'ouvrage, qui élargit le propos à une réflexion sur le pessimisme de l'anthropologie classique, laquelle, de Pascal à La Rochefoucauld et de La Fontaine à Saint-Simon, s'applique à révéler la bestialité de l'homme sous le masque de la civilité et la violence de l'amour-propre menaçant à chaque instant le pacte social. Aux monstres exhibés à Versailles répond désormais le monstre intérieur: relisant à point nommé quelques pages de Racine et de Perrault, Michel Jeanneret suggère que la sauvagerie qui sommeille en chaque être humain n'est pas niée par les œuvres classiques, mais tout à la fois révélée par elles et conjurée au moyen de l'harmonie des formes. C'est ainsi sur l'hypothèse d'une fonction apotropaïque de l'art classique, «voisinage de terreur et de style» (in fine) que s'achève ce livre majestueux, d'ombre et de lumière.

Le troisième et dernier temps de ce parcours à travers le XVII ${ }^{\mathrm{e}}$ siècle de Michel Jeanneret nous ramène à la puissance de liberté célébrée dans Éros rebelle, mais envisagée cette fois-ci sous un angle différent: celui de l'extravagance. Paru aux éditions Droz en 2018, J'aime ta joie parce qu'elle est folle. Ecrivains en fête (XVI' et $X V I I^{\circ}$ siècle), dont certains chapitres se nourrissent du travail éditorial consacré aux Historiettes de Tallemant des Réaux ${ }^{32}$, traverse notre pré-modernité en recueillant comme autant de précieuses pépites les diverses revendications, par l'œuvre ou par la vie, du droit à la gaieté exubérante. Après une première partie consacrée à quelques grandes figures de la Renaissance (Érasme, Rabelais, Folengo, Montaigne, Béroalde), puis un intermède qui rappelle la disparition progressive de la culture de la folie autorisée (fête des fous, confréries facétieuses, fonction de fou du roi, etc.), Michel Jeanneret étudie dans leur diversité les modes de survivance de l'excentricité au XVII siècle. Il se propose moins de revisiter certains textes que de nous présenter des milieux particuliers, des individus singuliers. Qu'il s'agisse des farceurs du Pont-Neuf ou des pitres de l'Hôtel de Bourgogne (Turlupin, Gros-Guillaume, Gaultier-Garguille, etc.), d'écrivains largement oubliés des non-spécialistes (SaintAmant, Dassoucy, Boisrobert, Voiture) ou de figures beaucoup plus canoniques (Scarron, Molière), c'est une galerie de portraits savoureux qui, parallèlement aux séries gravées des Gobbi et des Balli di Sfessania de Callot (pp. 124-128), se déploie sous nos yeux. Du Pont-Neuf au château de Versailles en passant par le cabinet de Richelieu et l'Hôtel de Rambouillet, le siècle apparait encore et toujours constellé de bouffonneries malgré la discipline et les interdits. Malgré l'image austère que certains continuent de promouvoir, guindés dans leur sérieux universitaire mais sans doute démunis face à la grande question de Tabarin: «Quelle différence il y a entre le nez et le cul?» (p. 141). On trouve dans ce dernier livre, sans doute plus léger et plus rapide que les précédents, non seulement un art de l'esquisse qui en rend la lecture extrêmement plaisante, mais aussi une dimension performative qui

(32) Tallemant des Réaux, Historiettes, Choix et présentation de M. Jeanneret. Établissement du texte par A. Adam. Notes d'A. Adam et de M. Jeanneret, Paris, Gallimard, «Folio classique», 2013. 
dépasse de beaucoup les questions strictement historiques et intellectuelles. La joie est ici partout, dans la parole et les actes des auteurs étudiés, dans l'écriture qui leur rend hommage et, par contagion, par bonheur, par Bruscambille! dans l'accueil que nous lui réservons.

FRÉDÉRIC TINGUELY

Université de Genève

\section{Métamorphoses de la folie}

Par deux fois, Michel Jeanneret a inscrit la folie dans ses titres. En 1978 paraît La lettre perdue, vertigineuse enquête consacrée aux rapports entre «écriture et folie dans l'œuvre de Nerval», ainsi que l'indique le sous-titre, où il évacue l'approche biographique et le déchiffrement psychiatrique pour «rendre à l'auteur son statut d'écrivain» ${ }^{33}$. Quarante ans plus tard, Michel célèbre dans son dernier livre, J'aime ta joie parce qu' elle est folle (2018), les «écrivains en fête», qui vivent la déraison comme une bombance, accueillent avec exubérance ce que Nietzsche nommait une «brave petite insanité», pour l'inoculer, à la façon des fous à la cour, dans la sociétée ${ }^{34}$. La folie n'est plus un abîme de la subjectivité, où l'écriture lutte contre les vertiges qui l'habitent, mais une arme aux mains de corsaires saltimbanques, qui bousculent l'ordre moral et les agélastes trop prompts à corseter leurs concitoyens. Du rêve d'Aurélia à la marotte, les fils de la folie se trament comme une belle étoffe bigarrée tout au long de l'œuvre critique de Michel Jeanneret, ou plutôt ils en constituent ce qu'Henry James aurait appelé le «motif dans le tapis», une clé secrète stimulant l'invention herméneutique.

C'est dans Poésie et tradition biblique (1969) que la folie fait sa première et timide apparition grâce au célèbre éloge d'Érasme, où sont couverts de quolibets les moines hypocrites ${ }^{35}$. Ce panégyrique soulève d'emblée la question fondamentale qui travaillera La lettre perdue - comment dire la folie? Jacques Derrida avait relevé le caractère paradoxal d'un tel projet, tout discours, en tant qu'acte logique, ne pouvant qu'aliéner l'aliénation, neutraliser le délire en le forçant dans les catégories de la raison: «la folie, c'est par essence ce qui ne se dit pas» ${ }^{36}$. Michel Jeanneret préfère livrer le logos à d'autres possibles qui l'«estrangent» à lui-même, ouvrant au cœur même du langage une «fêlure», une «profondeur où puisse s'immiscer la folie». L'espace de rencontre entre les mots et les maux de l'esprit a pour noms fiction, pathos. Tel serait le «privilège du poétique»: «capter l'indicible» dans les marges et les bordures d'une écriture seule capable de relever un défi impossible à la philosophie. La folie cherchant à se dire questionne l'essence même du travail littéraire, les pouvoirs de la fantaisie à faire et à défaire comme une Pénélope déchaînée - une méditation qui accompagna Michel toute sa vie.

Dépassant le paradoxe derridien, il met au jour ces formes d'ambiguïté discursives qui, loin d'épuiser le «mystère de la folie», l'épaississent, entraînent le lecteur

(33) M. Jeanneret, La Lettre perdue. Écriture et folie dans l'œuvre de Nerval, Paris, Flammarion, 1978.

(34) M. Jeanneret, J'aime ta joie parce qu'elle est folle. Écrivains en fête (XVI et XVII siècles), Genève, Droz, 2018, p. 11.

(35) M. Jeanneret, Poésie et tradition biblique au XVI siècle. Recherches stylistiques sur les paraphrases des psaumes de Marot à Malherbe, Paris, José Corti, 1969, p. 17.

(36) J. Derrida, «Cogito et histoire de la folie», in L'Ecriture et la différence, Paris, Seuil, 1967, p. 68, cité par M. Jeanneret, La Lettre perdue cit., p. 10. 
dans son inquiétante opacité. La Folie s'exprime, non pas en disloquant le style ni en fracturant le sens, mais en le rendant indécidable. Chez Érasme, l'unique et fantasque locutrice, Moira, parle pour se célébrer follement et montrer que la folie est partout. Or dit-elle follement un discours véridique, ou sérieusement des balivernes? Chez Montaigne, auquel Perpetuum mobile consacre de belles pages, la faille s'intériorise $^{37}$. S'il n'est «folie ny réverie» que ne produisent les esprits déréglés, le «je» divisé n'entend pas dresser mais mettre en rolle, contempler, les monstres fantasques qui l'agitent. La page se fait terrain vague pour accueillir le difforme, l'informe, des «fantasies irresolues» ${ }^{38}$. La mise au pas des extravagances de la psyché serait une duperie, à laquelle résiste tout aussi bien Michel Jeanneret que ces auteurs qui le fascinent, Montaigne et Nerval, vivant de la tension entre éclatement polyphonique et reconquête de l'unité. Si Montaigne loue ses chimères cognitives, semblables à des «rêves de malades» ${ }^{39}$, pour relativiser l'opposition entre santé et maladie, ressource féconde de l'écriture, Nerval questionne le partage entre norme et déviance dans sa célèbre lettre-préface, où il conteste à Alexandre Dumas le droit de statuer sur sa folie: «Moi, je m'étais brodé sur toutes les coutures. - Du moment que j’avais cru, il ne m'en coûtait pas plus d'avoir été prince, roi, mage, génie et même Dieu, la chaîne était brisée et marquait les beures pour des minutes» ${ }^{40}$. Dans le sillage figural des Essais, ce singulier «rapiecement» enrichi d'«ornements surnuméraires», Nerval utilise l'image de la broderie qui masque et exhibe les sutures d'un «je» disloqué.

Comme le montre Michel Jeanneret, c'est dans le rapport de son narrateur à l'autre, où s'abolissent les différences et se confondent les temporalités, dans les réseaux d'images obsédantes (errance, pénurie, absence, vaporisation de l'identité) et la charge affective des symboles que se joue une ambiguïté à la fois mortifère et salvatrice, jouissive et désespérée. «Tout est double mais tout est rêvé comme unique» ${ }^{41}$. Dans ce double jeu du discours de la folie sur la folie, il s'agit tout à la fois de la mimer et de la tenir à distance, de la revivre comme expérience et d'en faire l'investigation critique, de l'épouser, ce que Nerval ne fit jamais avec les femmes aimées, et de la maîtriser. Oscillant entre cette double fonction, mimétique et critique, le personnage-narrateur ne peut imposer un partage textuel trop rigoureux des êtres (Sylvie, Adrienne, Aurélie), des espaces (Paris - le Valois) et des temps (passé idyllique - présent dysphorique), sans menacer l'intégrité de son être singulier, qui lutte par l'indistinction contre les verdicts du réel. Michel Jeanneret peut ainsi déployer dans La Lettre perdue sa rare sensibilité aux stratégies formelles par lesquelles Nerval contient et ordonne ses débordements psychiques, «mais sans endiguer le délire pour en effacer toute trace». Loin d'imposer un modèle herméneutique binaire, où la folie comme refus s'opposerait au refus comme folie, Michel révèle la porosité des catégories et leur interpénétration, à la façon d'un thyrse baudelairien où le bâton, tuteur de vignes, s'hybriderait à la ligne arabesque et à ses prestigieuses pirouettes.

Si l'angoisse sourd dans les textes nervaliens et habite l'œuvre critique, qui se veut elle-même ordonnancement rassurant des errances serpentines d'une âme tourmentée, elle semble se dissiper dans les livres ultérieurs, qui des agapes symposiaques

(37) M. Jeanneret, Perpetuum mobile. Métamorphoses des corps et des oeuvres, de Vinci à Montaigne, Paris, Macula, 1997 (deuxième édition corrigée Genève, Droz, 2016).

(38) Montaigne, Les Essais, éd. P. Villey, Paris, Puf, 1965, I, 8, p. 32; I, 57, p. 317, cité par M. Jeanneret, Perpetuum mobile cit., pp. 111 et 112.

(39) Montaigne, Les Essais, I, 8, citation latine d'Horace: velut aegri somnia, vanae finguntur species.

(40) Gérard de Nerval, «Ä Alexandre Dumas», in Les Filles du feu, Les Chimères, éd. J. Bony, Paris, Garnier-Flammarion, 1994.

(41) M. Jeanneret, La Lettre perdue cit., p. 117. 
mènent aux rebellions d'Éros, puis à la figure du bouffon. Michel Jeanneret avait pensé intituler ce dernier livre «Que la joie demeure», avant de lui préférer la citation de Beaumarchais, «J'aime ta joie parce qu'elle est folle», où l'amour se lie à la joie d'une folie devenue toute festive. Dans ce livre, il écrit que l'allégresse «n'est pas une grâce tombée du ciel, mais une action, un combat, l'effort par lequel l'homme s'arrache à la prison, où souvent il s'est enfermé lui-même, pour prendre le large et se sentir élargi». Cultivons donc cette joie folle et qu'elle demeure, la joie infinie d'avoir connu et côtoyé Michel Jeanneret, d'avoir fendu avec lui les océans littéraires à la recherche de la dive bouteille, en compagnie de ses auteurs fétiches et un verre à la main. Comme l'écrit Nerval: «Ils reviendront, ces Dieux que tu pleures toujours! Le temps va ramener l'ordre des anciens jours; La terre a tressailli d'un souffle prophétique..... ${ }^{42}$. Et concluons avec Rabelais, qui, tout comme Michel Jeanneret, jugeait qu'on ne saurait jamais conclure: «Ergo gluc*. Ha, ha, ha, c'est parlé, cela» ${ }^{4}$.

DOMINIQUE BRANCHER

Université de Bâle

(42) Gérard de Nerval, «Delfica», vers 9-11, in Les Filles du Feu, Les Chimères.

(43) Rabelais, Gargantua, Lyon, Juste, 1535, ch. XVIII. 